hep-th/9908150

\title{
Gott Time Machines, BTZ Black Hole Formation, and Choptuik Scaling
}

\author{
Danny Birmingham \\ Department of Mathematical Physics \\ University College Dublin \\ Belfield, Dublin 4, Ireland \\ Siddhartha Sen[] \\ School of Mathematics \\ Trinity College Dublin \\ Dublin 2, Ireland
}

\begin{abstract}
We study the formation of BTZ black holes by the collision of point particles. It is shown that the Gott time machine, originally constructed for the case of vanishing cosmological constant, provides a precise mechanism for black hole formation. As a result, one obtains an exact analytic understanding of the Choptuik scaling.
\end{abstract}

August 1999

\footnotetext{
${ }^{1}$ E-mail: dannyb@pop3.ucd.ie

${ }^{2}$ E-mail: sen@maths.tcd.ie
} 
In [1], a precise mechanism was presented for the production of closed timelike curves (CTC's). In particular, the spacetime of two point particles with mass and boost parameters $\alpha$ and $\xi$, in $(2+1)$-dimensional spacetime with vanishing cosmological constant $\Lambda$, was shown to produce CTC's if the inequality, $\sin \frac{\alpha}{2} \cosh \xi>1$, is satisfied. In [2, 3], this Gott time machine was analysed in terms of the group theoretic approach to point particles [⿴囗十 . One essential feature of this approach is that the point particle spacetime is obtained via a suitable identification by an elliptic (timelike) generator of the isometry group. It was demonstrated in [2, 3] that the effective two-particle generator (the Gott time machine) becomes hyperbolic (spacelike) precisely when the Gott condition is satisfied. Various arguments were then presented to ensure that such CTC's do not arise in physically acceptable spacetimes [2, 3, 5, 6]. The possibility that black hole formation could provide an escape route from the CTC's was alluded to in [1]. However, the absence of a black hole spacetime with $\Lambda=0$ means that this route for chronology protection is unavailable.

It is noteworthy that in $(2+1)$-dimensional anti-de Sitter gravity $(\Lambda<0)$, we do indeed have the Bañados-Teitelboim-Zanelli (BTZ) black hole solution [7, 8], for a review see [9]. From our point of view, the most important aspect of this black hole is that it is defined by a hyperbolic isometry. One may choose a fundamental region of this hyperbolic isometry, and define the black hole spacetime by identification of the region's boundaries by the isometry. Equivalently, the black hole spacetime may be defined as the quotient of three-dimensional anti-de Sitter space $\left(\operatorname{adS}_{3}\right)$ by the cyclic group generated by the hyperbolic isometry 8]. In this paper, we show that the Gott time machine, suitably extended to the anti-de Sitter case, is precisely the mechanism for BTZ black hole formation in particle collisions. If the Gott condition is satisfied, the effective two-particle generator becomes hyperbolic. Thus, one can immediately identify the resulting quotient spacetime as the BTZ black hole. This understanding of black hole formation via the Gott time machine is based simply on the observation that the formation of the black hole is effected by the transition from two elliptic (particle) generators to a hyperbolic (black hole) generator.

Having understood BTZ black hole formation at the algebraic level, it is of interest to see if this sheds light on the origin of Choptuik scaling [10], for a review see [11]. Interest in this critical phenomenon is centered around the fact that the threshold for black hole formation in the space of initial data has a simple structure. In particular, the black hole parameters exhibit universal power-law scaling behaviour. We demonstrate that the natural order parameter for BTZ black hole formation is the trace of the associated generator. In this way, we gain an exact analytic understanding of Choptuik scaling in $2+1$ dimensions. It is then a simple matter to read off the Choptuik scaling, which has an exponent $\gamma=1 / 2$.

We recall [9] that $\operatorname{adS}_{3}$ can be viewed as the group manifold of $S L(2, \mathbf{R})$, with isometry group $(S L(2, \mathbf{R}) \times S L(2, \mathbf{R})) / Z_{2}$. Thus, for $\mathbf{X} \in S L(2, \mathbf{R})$, the isometry group acts by left and right multiplication, $\mathbf{X} \rightarrow \rho_{L} \mathbf{X} \rho_{R}$, with the identification $\left(\rho_{L}, \rho_{R}\right) \sim\left(-\rho_{L},-\rho_{R}\right)$. We shall use the equivalent $S U(1,1)$ notation instead of $S L(2, \mathbf{R})$; they are related by conjugation, which is given explicitly in [12].

As shown in 4 , the spacetime for a single static point particle with $\Lambda=0$ is obtained by removing a wedge of deficit angle $\alpha$, and identifying opposite sides of the wedge. The particle spacetime is defined via the rotation generator with angle $\alpha$,

$$
R(\alpha)=\left(\begin{array}{cc}
e^{-i \alpha / 2} & 0 \\
0 & e^{i \alpha / 2}
\end{array}\right)
$$


The mass $m$ of the particle is given by $\alpha=\pi m$, in units with $8 G=1$, and the resulting spacetime has a naked conical singularity.

A moving particle is obtained by boosting to the rest frame of the particle, rotating, and then boosting back. The corresponding boost matrix is

$$
B(\boldsymbol{\xi})=\left(\begin{array}{cc}
\cosh \frac{\xi}{2} & e^{-i \phi} \sinh \frac{\xi}{2} \\
e^{i \phi} \sinh \frac{\xi}{2} & \cosh \frac{\xi}{2}
\end{array}\right),
$$

where $\boldsymbol{\xi}$ is the boost vector with $\xi=|\boldsymbol{\xi}|$, and $\phi$ is the polar angle. Thus, the generator for a moving particle is

$$
T=B(\boldsymbol{\xi}) R(\alpha) B^{-1}(\boldsymbol{\xi})
$$

where

$$
\begin{aligned}
& T_{11}=e^{-i \alpha / 2}\left[\cosh ^{2} \frac{\xi}{2}-e^{i \alpha} \sinh ^{2} \frac{\xi}{2}\right], \\
& T_{12}=2 i \sin \frac{\alpha}{2} e^{-i \phi} \cosh \frac{\xi}{2} \sinh \frac{\xi}{2} \\
& T_{21}=T_{12}^{*}, T_{22}=T_{11}^{*} .
\end{aligned}
$$

Since our considerations rest in anti-de Sitter space, we simply note that the static and moving particle spacetimes are defined in an analogous fashion, with left and right generators. Particle spacetimes for non-zero cosmological constant have been considered in [13].

To construct the Gott time machine, we consider a two-body collision process, with particles labelled by $A$ and $B$. The effective two-particle generator is then the product [2, 3, 1 , namely $T^{G}=T_{B} T_{A}$. The central object of interest to us is the trace of this generator. Using (田), it is straightforward to compute

$$
\begin{aligned}
\frac{1}{2} \operatorname{Tr} T^{G} & =\cos \frac{\alpha_{A}}{2} \cos \frac{\alpha_{B}}{2}+\sin \frac{\alpha_{A}}{2} \sin \frac{\alpha_{B}}{2} \\
& -\sin \frac{\alpha_{A}}{2} \sin \frac{\alpha_{B}}{2}\left[\cosh ^{2}\left(\frac{\xi_{A}+\xi_{B}}{2}\right)+\cosh ^{2}\left(\frac{\xi_{A}-\xi_{B}}{2}\right)\right] \\
& +\sin \frac{\alpha_{A}}{2} \sin \frac{\alpha_{B}}{2} \cos \left(\phi_{A}-\phi_{B}\right)\left[\cosh ^{2}\left(\frac{\xi_{A}+\xi_{B}}{2}\right)-\cosh ^{2}\left(\frac{\xi_{A}-\xi_{B}}{2}\right)\right] .
\end{aligned}
$$

The original Gott time machine is recovered by choosing particles with equal masses, and equal and opposite boosts, namely $\alpha_{A}=\alpha_{B}=\alpha, \xi_{A}=\xi_{B}=\xi, \phi_{A}-\phi_{B}=\pi$. We find

$$
\frac{1}{2} \operatorname{Tr} T^{G}=1-2 \sin ^{2} \frac{\alpha}{2} \cosh ^{2} \xi
$$

The significance of this result can be appreciated by recalling that the isometries of adS $\mathrm{S}_{3}$ are classified according to the value of their trace. We have

$|\operatorname{Tr} T|<2$, Elliptic,

$|\operatorname{Tr} T|=2$, Parabolic,

$|\operatorname{Tr} T|>2$, Hyperbolic. 
Thus, when the Gott condition is satisfied, we have $\sin ^{2} \frac{\alpha}{2} \cosh ^{2} \xi>1$, and thus $T^{G}$ is a hyperbolic generator, and when $\sin ^{2} \frac{\alpha}{2} \cosh ^{2} \xi<1$, we have an elliptic generator.

Armed with this observation, we are now in a position to discuss the implications of the Gott time machine for black hole formation. First, we recall that the conventional mass parameter of the BTZ black hole is denoted by $M$, while the point particle mass $m$ is related by $m=$ $2(1-\sqrt{-M})$. As a result, the point particle mass spectrum is $-1<M<0$, while the black hole mass spectrum is $M \geq 0$, with $M=-1$ corresponding to $\operatorname{adS}_{3}$.

Let us first consider the static black hole case, in which the left and right generators are taken to be equal [9]. We recall also that the isometries of $\mathrm{adS}_{3}$ are subject to the identification $\left(\rho_{L}, \rho_{R}\right) \sim\left(-\rho_{L},-\rho_{R}\right)$. Thus, we may take $\rho_{L}=\rho_{R}=-T^{G} \equiv \rho$. The BTZ black hole is defined as the quotient of $\operatorname{adS}_{3}$ by a cyclic group with a single hyperbolic generator [8]. If the Gott condition is satisfied, then as we have seen $T^{G}$ is a hyperbolic generator. Thus, the Gott time machine results in BTZ black hole formation. The black hole mass is then given by [9, 12]

$$
\frac{1}{2} \operatorname{Tr} \rho=\cosh \pi \sqrt{M}=-1+2 \sin ^{2} \frac{\alpha}{2} \cosh ^{2} \xi \equiv p
$$

where $p \geq 1$.

It is important to consider the defining equation of this process, namely

$$
T_{B} T_{A}=T^{G}
$$

On the left-hand side, we have the input data given by the particle mass and boost parameters $\alpha$ and $\xi$. The incoming particles $A$ and $B$ have been set up in a symmetrical way, with equal mass and boost parameters, and the timelike geodesics representing their worldlines will intersect at a given time, say $t=0$. Thus, we may regard this as the time of collision of the two particles. The product of the particle generators represents the effective generator of the system at this time 114. As we have seen, the effective generator at the time of collision is hyperbolic if the Gott condition is satisfied. We may then interpret equation (9) as defining the formation of a BTZ black hole at time $t=0$, with the value of the black hole mass fixed by the input parameters $\alpha$ and $\xi$. Thus, equation (9) encodes dynamical information. However, the precise details of the motion of the particles corresponding to the generators $T_{A}$ and $T_{B}$ prior to the collision, as well as the motion after collision may also be studied. Indeed, this analysis has been performed for massless particles in [14, 15].

We see from (8) that the natural order parameter for black hole formation in $(2+1)$ dimensional anti-de Sitter gravity is the trace of the generator. This takes a critical value at the threshold for black hole formation, corresponding to the critical value of the parameter $p_{*}=1$. Clearly, $p=p_{*}$ corresponds to the black hole vacuum $M=0$, where the Gott generator is parabolic. Since the parameter $p$ depends on the initial data $\alpha$ and $\xi$, we can read off the critical boost $\xi$ for any given mass $\alpha$. As an example, taking $\alpha=\pi / 3$, we have black hole formation when $\cosh \xi \geq 2$. We have

$$
\pi \sqrt{M}=\operatorname{arccosh} p=\ln \left[p+\sqrt{p^{2}-1}\right] .
$$

We stress that the above expression is an exact analytic formula for the formation of a BTZ black hole in terms of the input (initial) parameters $\alpha$ and $\xi$, equivalently $p$. From this, we can immediately determine the Choptuik scaling, by studying the behaviour near $p_{*}$. The mass 
$M$ and horizon length $r_{+}$are related by $\sqrt{M}=r_{+} / l$, where $l$ is the scale of $\operatorname{adS}_{3}$. Writing $p=p_{*}+\epsilon$, we find to leading order

$$
\frac{r_{+}}{l}=\frac{\sqrt{2}}{\pi}\left(p-p_{*}\right)^{1 / 2}
$$

Thus, we observe a scaling factor of $\gamma=1 / 2$. Note that this is indeed a universal scaling since BTZ formation always requires a hyperbolic generator. The universal scaling value of $1 / 2$ is simply a consequence of the fact that the mass depends on the inverse cosh function. We also note that the derivative of the order parameter diverges at the critical value $p_{*}$.

If the Gott condition is not satisfied, then one has an effective particle spacetime with an elliptic generator, whose effective deficit angle is denoted by $\alpha_{\text {eff }}$. This can be obtained by continuation of (8) to negative $M$ values. We find,

$$
\frac{1}{2} \operatorname{Tr} \rho=\cos \pi \sqrt{-M}=-1+2 \sin ^{2} \frac{\alpha}{2} \cosh ^{2} \xi \equiv p,
$$

where now $p<p_{*}$. Once again, we have an exact analytic expression for the mass parameter on the other side of the transition, and of course the Choptuik scaling exponent is again $\gamma=1 / 2$, with

$$
\alpha_{\mathrm{eff}}=2 \pi-2 \sqrt{2}\left(p_{*}-p\right)^{1 / 2} .
$$

While the Choptuik scaling is evident near the black hole threshold, we stress that in this model we have recourse to the exact analytic expression (10).

To obtain the spinning BTZ black hole, we simply need to have independent left and right generators, and these are also given in terms of the Gott generators. Taking $\alpha_{A}=\alpha_{B}=$ $\alpha, \phi_{A}-\phi_{B}=0$, we find the left generator $\rho_{L}=-T^{G}$, with

$$
\frac{1}{2} \operatorname{Tr} \rho_{L}=\cosh \left(\frac{\pi}{l}\left(r_{+}-r_{-}\right)\right)=-1+2 \sin ^{2} \frac{\alpha}{2} \cosh ^{2}\left(\frac{\xi_{A}-\xi_{B}}{2}\right) \equiv \tilde{p} .
$$

For the right generator, we choose $\alpha_{A}=\alpha_{B}=\alpha, \phi_{A}-\phi_{B}=\pi$, with $\rho_{R}=-T^{G}$, leading to

$$
\frac{1}{2} \operatorname{Tr} \rho_{R}=\cosh \left(\frac{\pi}{l}\left(r_{+}+r_{-}\right)\right)=-1+2 \sin ^{2} \frac{\alpha}{2} \cosh ^{2}\left(\frac{\xi_{A}+\xi_{B}}{2}\right) \equiv p .
$$

The input data for particles $A$ and $B$ is no longer symmetric, but nevertheless $\rho_{L}$ and $\rho_{R}$ both become hyperbolic if the input parameters $\alpha, \xi_{A}, \xi_{B}$ satisfy the appropriate Gott conditions. In the above, we have introduced the parameters $r_{ \pm}$which denote the locations of the inner and outer horizons [9]. They are related to the mass $M$ and angular momentum $J$ by $M=$ $\left(r_{+}^{2}+r_{-}^{2}\right) / l^{2}, J=2 r_{+} r_{-} / l$. In this case, we obtain Choptuik scaling for both mass and angular momentum in the form

$$
\begin{aligned}
& \frac{r_{+}-r_{-}}{l}=\frac{\sqrt{2}}{\pi}\left(\tilde{p}-\tilde{p}_{*}\right)^{1 / 2}, \\
& \frac{r_{+}+r_{-}}{l}=\frac{\sqrt{2}}{\pi}\left(p-p_{*}\right)^{1 / 2} .
\end{aligned}
$$

As pointed out in [16], the Euclidean BTZ black hole is a geometrically finite Kleinian manifold with the topology of a solid torus. In essence, this allows one to invoke a theorem of 
Sullivan [17, 18 to establish a no hair theorem for the Euclidean black hole. As a result, the black hole and its hyperbolic generators are described by at most two parameters. Assuming that the continuation to Lorentzian signature does not induce additional parameters, one concludes that the BTZ black hole is described by mass and angular momentum only. One can consider many-body extensions of the Gott time machine. By the above reasoning, these will lead to the formation of a spinning BTZ black hole, if there are Gott conditions which produce independent left and right hyperbolic generators.

In conclusion, we have shown that the original Gott time machine provides a precise mechanism for BTZ black hole formation in particle collisions. The problems of CTC's and chronology protection [19] are overcome by the creation of the black hole horizon, as soon as the Gott condition is satisfied. This is indeed a satisfying scenario. We mention that the recent study of BTZ black hole formation from massless particle collisions [14, 15] is based on the lightlike analogue of the Gott time machine [20], and thus is also guaranteed to produce the hyperbolic generator necessary for BTZ formation. The holographic description of this creation process has been investigated within the adS/CFT correspondence in [21]. A Gott time machine in anti-de Sitter space was also studied in [22]. By definition, the BTZ black hole is defined as the quotient spacetime of a hyperbolic generator. Thus, irrespective of which type of matter is used to produce such a black hole, the ultimate result is that the mass of the black hole is defined via the trace of the hyperbolic generator. Hence, Choptuik scaling with a critical exponent $\gamma=1 / 2$ will always be present. Indeed, this exponent was observed for collapsing dust shells in 23.

\section{Acknowledgements}

This work is part of a project supported by Enterprise Ireland Basic Research Grant SC/98/741. D.B. would like to thank the Theory Division at CERN for hospitality, and Luis Alvarez-Gaumé for valuable discussions.

\section{References}

[1] J.R. Gott, Phys. Rev. Lett. 66 (1991) 1126.

[2] S.M. Carroll, E. Farhi, and A.H. Guth, Phys. Rev. Lett. 68 (1992) 263.

[3] S. Deser, R. Jackiw, and G. 't Hooft, Phys. Rev. Lett. 68 (1992) 267.

[4] S. Deser, R. Jackiw, and G. 't Hooft, Ann. Phys. (N.Y.) 152 (1984) 220.

[5] S.M. Carroll, E. Farhi, A.H. Guth, and K.D. Olum, Phys. Rev. D50 (1994) 6190; grqc/9404065.

[6] P. Menotti and D. Seminara, Ann. Phys. (N.Y.) 240 (1995) 203; gr-qc/9406016.

[7] M. Bañados, C. Teitelboim, and J. Zanelli, Phys. Rev. Lett. 69 (1992) 1849; hepth/9204099.

[8] M. Bañados, M.Henneaux, C. Teitelboim, and J. Zanelli, Phys. Rev. D48 (1993) 1506; gr-qc/9302012.

[9] S. Carlip, Class. Quantum. Grav. 12 (1995) 2853; gr-qc/9506079. 
[10] M.W. Choptuik, Phys. Rev. Lett. 70 (1993) 9.

[11] C. Gundlach, Adv. Theor. Math. Phys. 2 (1998) 1; gr-qc/9712084.

[12] A.R. Steif, Phys. Rev. D53 (1996) 5527; gr-qc/9511053.

[13] S. Deser and R. Jackiw, Ann. Phys. (N.Y.) 153 (1984) 405.

[14] H.-J. Matschull, Class. Quantum Grav. 16 (1999) 1069; gr-qc/9809087.

[15] S. Holst and H.-J. Matschull, The Anti-de Sitter Gott Universe: A Rotating BTZ Wormhole, gr-qc/9905030.

[16] D. Birmingham, C. Kennedy, S. Sen, and A. Wilkins, Phys. Rev. Lett. 82 (1999) 4164; hep-th/9812206.

[17] D. Sullivan, in Proceedings of the 1978 Stony Brook Conference on Riemann Surfaces and Related Topics, edited by I. Kra and B. Maskit, Annals of Mathematics Studies No. 97, Princeton University Press, Princeton, New Jersey, 1981.

[18] C. McMullen, Bull. Am. Math. Soc. 27 (1992) 207; Invent. Math. 99 (1990) 425.

[19] S.W. Hawking, Phys. Rev. D 46 (1992) 603.

[20] S. Deser and A.R. Steif, Class. Quantum Grav. 9 (1992) L153; hep-th/9208018.

[21] V. Balasubramanian and S.F. Ross, Holographic Particle Detection, hep-th/9906226.

[22] S. Holst, Gen. Rel. Gravit. 28 (1996) 387; gr-qc/9501010.

[23] Y. Peleg and A.R. Steif, Phys. Rev. D51 (1995) R3992; gr-qc/9412023. 